\title{
A, Nematostella
}

JPRED

QUERY : APTTPSAVHGEEDKNKPHRKHEERERHHGRHGNSKKHRKHDEEDEPENERDFFGEPRSPAT : QUERY

UniRef90 A7RTM2 : APTTPSAVHGEEDKNKPHRKHEERERHHGRHGNSKKHRKHDEEDEPENERDFFGEPRSPAT :

OrigSeq $\quad:$ : APTTPSAVHGEEDKNKPHRKHEERERHHGRHGNSKKHRKHDEEDEPENERDFFGEPRSPAT : OrigSeq

Jnet

jhmm

jpssm

Lupas 14

Lupas 21

Lupas 28

Jnet_25

Jnet 5

Jnet ${ }^{-} 0$

Jnet ${ }^{-} \operatorname{Re}$

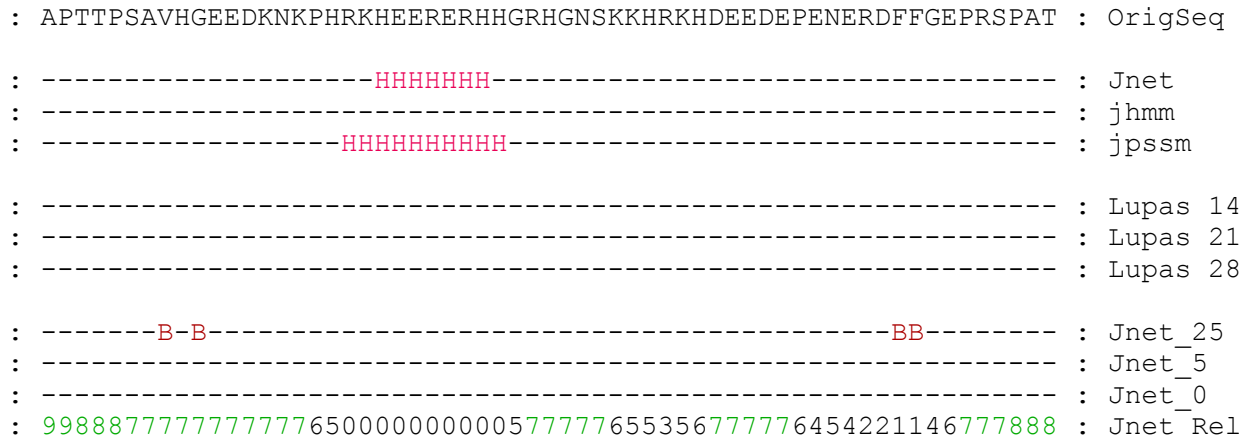

PSIPRED

$\mathrm{AA}$ : Target sequence

Conf: 998888513764579965125888752068899620146788888763223458999999

P red： C C C C C C C C C C C C C C C C C 目目目田直目 C C C C C C C C C C C C C C C C C C C C C C C C C C C C C C

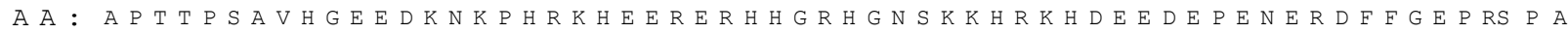

\section{B, Caenorhabditis e.}

JPRED

QUERY $\quad:$ SSSSTSSESDEDEDKNIKELRVDIEPIIDEPASFYRHDKLIQSPEVERSASSVFQP : QUERY

UniRef90 Q10651 : -----------KNIKELRVDIEPIIDEPASFYRHDKLIQSPEVERSASSVFQP :

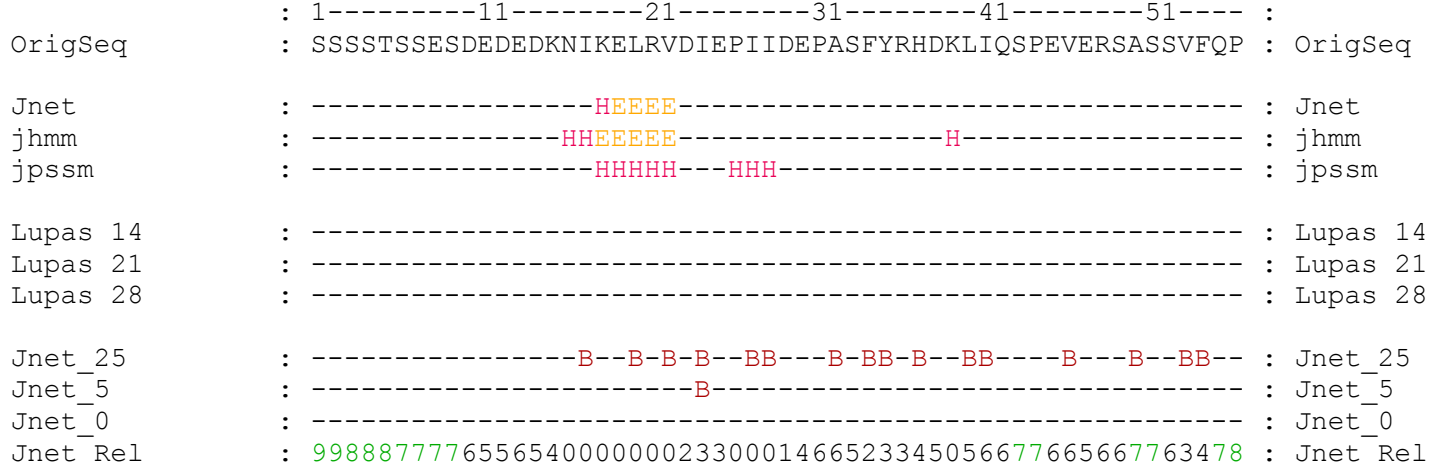

PSIPRED

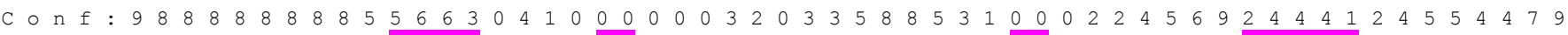

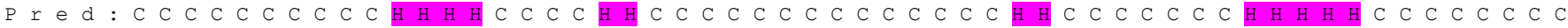

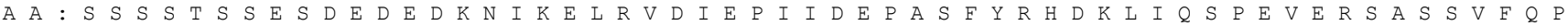

\section{C, Apis}

JPRED

QUERY

UniRef90 UPI00015B49D1

Origseq

Jnet

jhmm

jpssm

Lupas 14

Lupas 21

Lupas 28

Jnet 25

Jnet_5

Jnet 0

Jnet ${ }^{-}$Rel
LRTEQAQQEESDS INDNKDIQQQPEQPAAMHSEGLVRAAHSLTHDISHSEPGYSVRREMYHRESRS : QUERY

: - -

: 1--------11--------21--------31--------41--------51--------61---- :

: LRTEQAQQEESDS INDNKDIQQQPEQPAAMHSEGLVRAAHSLTHDISHSEPGYSVRREMYHRESRS : OrigSeq

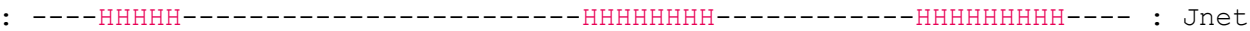

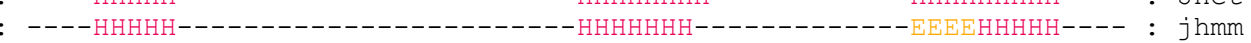

: ----нннннн--_-_-_-_-

: -
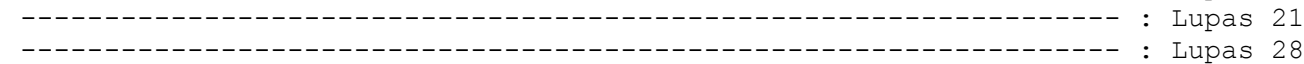

:
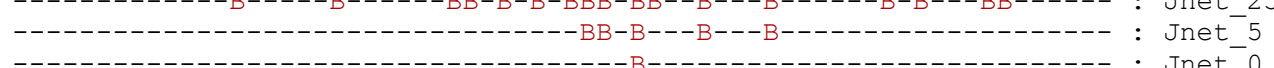

997367764046777776543234356654564166665300036788776630000867621799 : Jnet ${ }^{-} \operatorname{Rel}$ 
PSIPRED

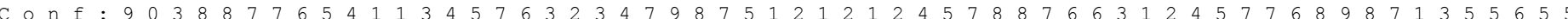
$\mathrm{P} r \mathrm{e} d \mathrm{~d}: \mathrm{C}$ C $\mathrm{H}$ H H H H H H H H C C C C C C C C C C C C C C C C

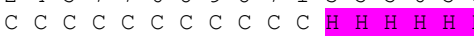

C $\circ$ n f : $: 4 \begin{array}{lllllllll}2 & 1 & 0 & 2 & 7 & 9\end{array}$

$\mathrm{P} r \mathrm{e} d: \mathrm{H}$ H $\mathrm{H}$ C $\mathrm{C}$ C $\mathrm{C}$

A A : Y H R S R S

\section{D, Drosophila wil.}

JPRED

QUERY : ATVSSTKVQTALPTVDDDAVQRAVEDVAAAVAHQEAEPQVQHFMTHDLGHRESSFSLRREFAHSHATKEGRN : QUERY

UniRef90 Q0IFC5 : ATISNSPQSTALPTVDDEAVQRAVEEVAAAVAHQEAEPKMQHILSHDTGHSEPTFSVRREIYG-------- : UniRef90 Q0IFC5

UniRef90 Q7QCS9 :-----SPQPTALPTVDDEAVQRAVEEVAAAVAHQEAEPKMQHVLAHDIGHGEP---------------- : UniRef90 Q7QCS9

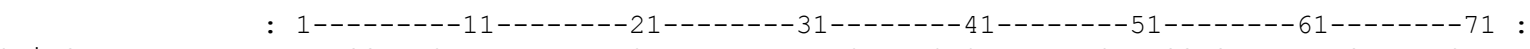

OrigSeq : ATVSSTKVQTALPTVDDDAVQRAVEDVAAAVAHQEAEPQVQHFMTHDLGHRESSFSLRREFAHSHATKEGRN : OrigSeq

Jnet

$j$ jmm

jpssm

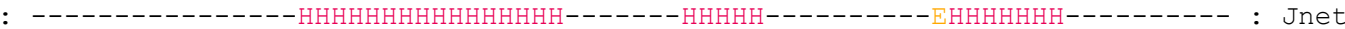
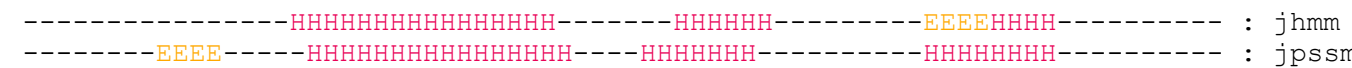

Lupas 14

Lupas 21

Lupas 28

Jnet 25

Jnet_5

Jnet ${ }^{-} 0$

Jnet $\operatorname{Rel}$

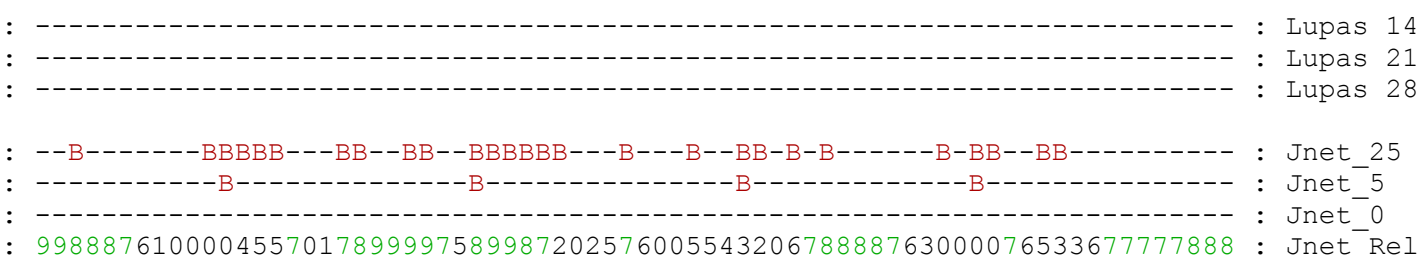

PSIPRED

C $\circ \mathrm{nf}: \begin{array}{lllllllllllllllllllllllllllllllllllllllllllllllllllllllll} & 4 & 3 & 6 & 6 & 5 & 0 & 0 & 4 & 6 & 7 & 6 & 6 & 7 & 6 & 9 & 9 & 9 & 9 & 9 & 9 & 9 & 9 & 9 & 9 & 9 & 9 & 9 & 6 & 4 & 1 & 3 & 1 & 1 & 2 & 1 & 1 & 3 & 5 & 5 & 6 & 5 & 4 & 1 & 2 & 6 & 8 & 8 & 8 & 8 & 7 & 3 & 1 & 3 & 4 & 3 & 5\end{array}$

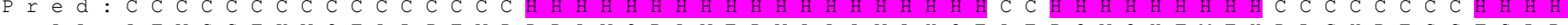

A A : A T V S S T K V Q T A L P T V D D D A V Q R A V E D V A A A V A H Q E A E P Q V Q H F M T H D L G H R E S S F S L R R

C $\circ$ n f : $\begin{array}{llllllllllll}4 & 2 & 1 & 4 & 5 & 6 & 3 & 2 & 4 & 6 & 9 & 9\end{array}$

Pre d : H H H C C C C C C C C C C

A A : E F A H S H T K E G R N

\section{E, Loligo}

JPRED

QUERY : TEARPEQQEDIKTPGFDSETFEDERPAIAEQTIHDLPNHRKKGYIAHVQQQPMIADEVSLDNLYANSHAN : QUERY

UniRef90 Q06BR2 : TEARPEQQEDIKTPGFDSETFEDERPAIAEQTIHDLPNHRKKGYIAHVQQQPMIADEVSLDNLYANSHAN : UnIREf90 Q06BR2

OrigSeq : TEARPEQQEDIKTPGFDSETFEDERPAIAEQTIHDLPNHRKKGYIAHVQQQPMIADEVSLDNLYANSHAN : OrigSEq

Jnet

jhmm

jpssm

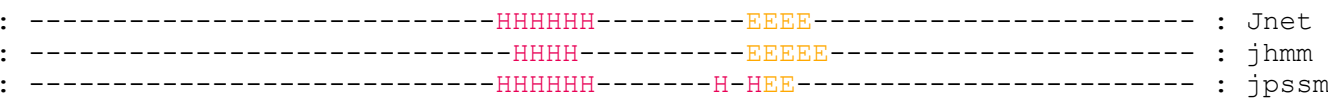

Lupas 14

Lupas 21

Lupas 28

Jnet 25

Jnet 5

Jnet 0

Jnet $\mathrm{Re}]$

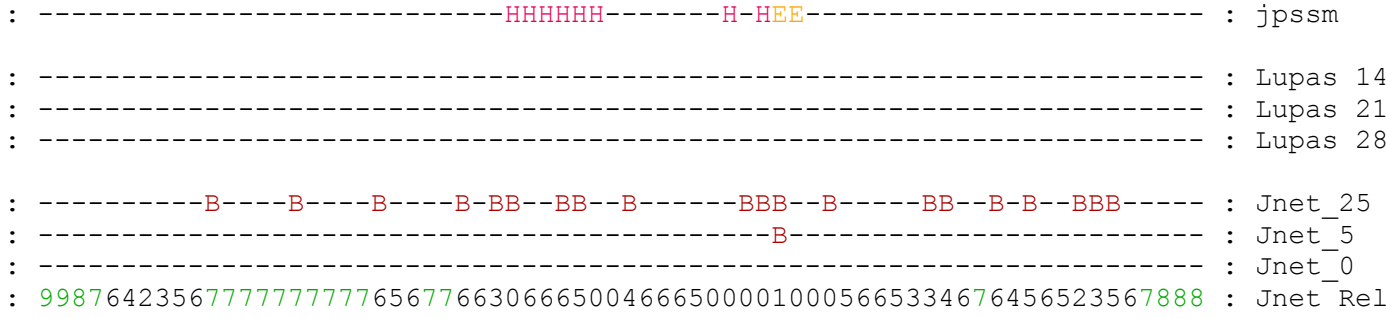

PSIPRED

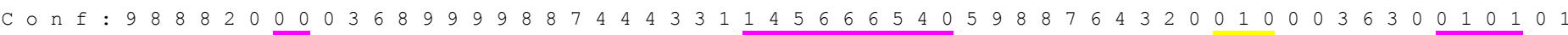
$\mathrm{P} r \mathrm{e} d \mathrm{a}$ C C C C C C H H C C C C C C C C C C C C C C C C C H H H H H H H H H C C C C C C C C C C E E E C C C C C C H H H H C C A A : TE A R P Q Q E D I K T P G D S E T F E D E R P A I A E Q T I H D L P N H R K G Y I A H $Q Q Q P M I A D E V S$

C $\circ$ n f : $: \begin{array}{lllllllllllllllllllll}0 & 0 & 1 & 1 & 1 & 1 & 3 & 5 & 8 & 9\end{array}$

$\mathrm{P} r \mathrm{e} \mathrm{d}: \mathrm{CHH}$ H H H C C C C C

A A : L D N L Y A N S H A N 


\section{F, Homo}

JPRED

QUERY

UniRef90 Q4S0J4

: ANTENEVEPVDARPAADRGLTTRPGSGLTNIKTEEISEVKMDAEFRHDSGYEVHHQKLVFFAEDVGSNKG : QUERY

UniRef90 Q4S0J4

UniRef90 073683

UniRef90 073683

UniRef90 UPI00016E77F7 : ANTENHVEPVDARPIPDRGLPTRP---------EEMPEVRTETDKRQSAGYEVYHQKLVFFADDVGSNKG :

UniRef90 UPI00016E77F7

UniRef90 Q5XIY5

UniRef90 Q5XIY5

UniRef90 Q8UUI8

UniRef90 Q8UUI8

UniRef90 UPI00016E7DA8 : ---------------------VTGKSMEAIPDMRMETEDRQSTEYEVHHQKLVFFAEDVGSNKG :

UniRef90 UPI00016E7DA8

UniRef90 Q3MQ25

UniRef90 Q3MQ25

UniRef90 Q28053

UniRef90 Q28053

: ANTENHVEPVDARPVPDRGLPTRPGKGVSALKPEEMPOVRMETDEROSAGYEVYHEKLVFFAEDVGSNKG :

: -NTENQVEPVDSRPTFERGVPTRPVTGKS---MEAVPELRMETEDRQSTEYEVHHQKLVFFAEDVGSNKG :

: ANTDNHVEPVDARPIPERGLPTRP----------EIPKVRLDIEERHNAGYDVRDKRLMFLAEDMGSNKG :

: -NTHNQVEPVDARPVPDLDLATRPVSGL---KPDDIPELRMEAEERHS---EVYHQKLVFFAEDVSSNKG :

Origseq

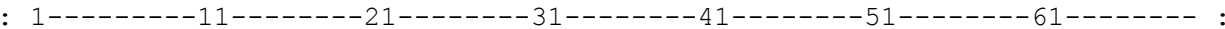

Jnet

jhmm

jpssm

Lupas 14

Lupas 21

Lupas 28

Jnet 25

Jnet_5

Jnet 0

Jnet Rel

: ANTENEVEPVDARPAADRGLTTRPGSGLTNIKTEEISEVKMDAEFRHDSGYEVHHQKLVFFAEDVGSNKG : OrigSeq

PSIPRED

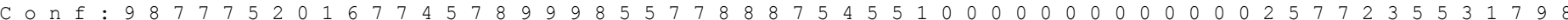

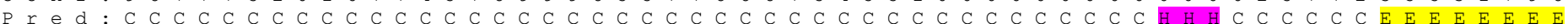
A A : A N T E N E V E P V D A R P A A D R G L T T R P G S G L T N I K T E E I S E V K M D A E F R

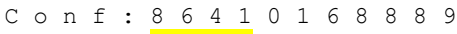

P r e d : F F E F C C C C C C C

A A : F F A E D V G S N K G

\section{Key for JPRED:}

Colour code for alignment:

Blue - Complete identity at a position

Shades of red - The more red a position is, the higher the level of conservation of chemical properties of the amino acids

Jnet

- Final secondary structure prediction for query $\mathrm{H}: \alpha$-helix; E: $\beta$-strand

jalign - Jnet alignment prediction

jhmm - Jnet hmm profile prediction

jpssm - Jnet PSIBLAST pssm profile prediction

Lupas - Lupas Coil prediction (window size of 14, 21 and 28)

Note on coiled coil predictions - = less than 50\% probability

$\mathrm{c}=$ between $50 \%$ and $90 \%$ probability

$\mathrm{C}=$ greater than $90 \%$ probability

Jnet 25 - Jnet prediction of burial, less than 25\% solvent accesibility

Jnet_5 - Jnet prediction of burial, less than 5\% exposure

Jnet $0 \quad$ - Jnet prediction of burial, 0\% exposure

Jnet Rel - Jnet reliability of prediction accuracy, ranges from 0 to 9 , bigger is better.

\section{Key for PSIPRED:}

Conf: Confidence (0=low, $9=$ high)

Pred: Predicted secondary structure (H=helix, E=strand, C=coil)

AA: Target sequence 